Haley Calcagnotto

Ana LÚCia LetTi MÜller ${ }^{1}$

Julio Cesar Loguercio Lette ${ }^{2}$

Maria Teresa Vieiro SanseVerinO ${ }^{2}$

KeLLI WAgner Gomes ${ }^{3}$

José ANTONIO de AZEVEDo Magalhães ${ }^{1,4}$

\title{
Fatores associados à mortalidade em recém-nascidos com gastrosquise
}

\author{
Associated factors for perinatal mortality in gastroschisis
}

Artigo Original

\section{Palavras-chave \\ Gastrosquise \\ Mortalidade perinatal Recém-nascido de baixo peso \\ Prematuro \\ Sepse \\ Keywords}

Gastroschisis Perinatal mortality Infant, low birth weight Infant, premature

Sepsis

\section{Resumo}

OBJETIVO: Analisar a taxa de mortalidade perinatal dos casos de gastrosquise e os possíveis fatores associados. MÉTODOS: Foi realizado estudo de coorte retrospectivo entre 1992 e 2012 . Foram incluídos todos os casos de gastrosquise nascidos no Hospital de Clínicas de Porto Alegre (HCPA) naquele período. $O$ diagnóstico de gastrosquise foi obtido por meio do exame ultrassonográfico morfológico ou pelo exame clínico ao nascimento nos casos desconhecidos no pré-natal. As variáveis de nascimento (peso ao nascer, idade gestacional e escore de Apgar, modo de parto, tipo de gastrosquise e anomalias associadas) e cirúrgicas (tipo de fechamento cirúrgico, reintervenção e sepsel foram comparadas entre os casos sobreviventes e os óbitos. Os resultados desta comparação foram analisados de acordo com o tipo de variável por meio de testes paramétricos e não paramétricos (MannWhitney ou teste $t$ de Student, $\chi^{2}$ ou teste exato de Fisher) sendo considerado o nível de significância de $5 \%(p=0,05)$. RESULTADOS: Foram incluídos 64 recém-nascidos com gastrosquise, 59 deles (92,2\%) diagnosticados durante o pré-natal. Vinte e seis casos $(40,6 \%)$ tinham somente intestino exposto, classificados como gastrosquise simples, 22 tinham intestino e estômago $(34,4 \%)$ e 16 tinham intestino e outros órgãos $(25 \%)$, totalizando 38 casos de gastrosquise complexa. O reparo cirúrgico primário foi realizado em 44 casos $(68,8 \%$ ). A mortalidade foi de 23,4\% (15 mortes). Os casos de óbito tinham peso ao nascer ( $p=0,001)$, escore de Apgar ( $p=0,03)$ e idade gestacional $(p=0,03)$ significativamente menores que os sobreviventes. Não houve diferença no modo de parto $(p=0,8)$ e, com relação ao conteúdo eviscerado, não houve diferença entre os casos de gastrosquise simples e complexa $(p=0,06)$. A mortalidade foi significativamente mais elevada entre os casos que necessitaram de reintervenção $(p=0,001)$ e com sepse $(p=0,008)$. CONCLUSÃO: A mortalidade perinatal da gastrosquise parece depender principalmente da prematuridade, baixo peso e complicações cirúrgicas.

\section{Abstract}

PURPOSE: To analyze the perinatal mortality rate in cases of gastroschisis and possible associated factors. METHODS: A retrospective cohort study was conducted between 1992 and 2012 . All cases of gastroschisis born in Hospital de Clínicas de Porto Alegre (HCPA) during that period were included. The diagnosis of gastroschisis was obtained by morphological ultrasound examination or clinical examination at birth in prenatally unknown cases. The variables of birth (birthweight, gestational age and Apgar score, mode of delivery, type of gastroschisis and associated anomalies) and the surgical ones (type of surgical closure, reintervention and sepsis) were compared between surviving cases and deaths. The results of this comparison were analyzed according to the type of variable using parametric and non-parametric tests (Mann-Whitney or Student's t-test, $\chi^{2}$ or Fisher's exact test), with the level of significance set at $5 \%$ $(p=0.05)$. RESULTS: Sixty-four newborns with gastroschisis were included, 59 of them (92.2\%) diagnosed during the prenatal period. Twenty-six patients (40.6\%) had only exposed intestines, classified as simple gastroschisis, 22 had exposure of the intestines and stomach (34.4\%) and 16 had exposure of the intestine and other organs (25\%), for a total of 38 cases of complex gastroschisis. Primary surgical repair was performed in 44 cases $(68.8 \%)$. The mortality rate was $23.4 \%$ (15 deaths). Babies who died had significantly lower birth weight ( $p=0.001)$, gestational age
Correspondência

Haley Calcagnotito

Rua Ramiro Barcelos, 2.350/1.125 - Rio Branco

(EP: $90035-903$

Porto Alegre (RS), Brasil

Recebido

$25 / 11 / 2013$
Serviço de Ginecologia e Obstetrícia, Setor de Medicina Fetal e Serviço de Genética Médica do Hospital de Clínicas de Porto Alegre HCPA - Porto Alegre (RS), Brasil.

'Grupo de Medicina Fetal do Serviço de Ginecologia e Obstetrícia, Hospital de Clínicas de Porto Alegre - HCPA - Porto Alegre (RS), Brasil. ${ }^{2}$ Serviço de Genética e do Grupo de Medicina Fetal, Hospital de Clínicas de Porto Alegre - HCPA - Porto Alegre (RS), Brasil.

${ }^{3}$ Residência Médica, Hospital de Clínicas de Porto Alegre - HCPA - Porto Alegre (RS), Brasil.

${ }_{4}^{4}$ Departamento de Ginecologia e Obstetrícia, Faculdade de Medicina, Universidade Federal do Rio Grande do Sul - UFRGS -

Porto Alegre (RS), Brasil.

Conflito de interesses: não há 
$(p=0.03)$ and Apgar score $(p=0.03)$ than survivors. There was no difference in mode of delivery $(p=0.8)$ and, with respect to gut contents, there was no difference between the cases of simple and complex gastroschisis $(p=0.06)$. Mortality was significantly higher in patients with sepsis ( $p=0.008)$ and reintervention ( $p=0.001)$. CONCLUSION: in the present study, perinatal mortality due to gastroschisis seemed to depend mainly on prematurity, low birth weight, and surgical complications.

\section{Introdução}

Gastrosquise é uma malformação congênita caracterizada por um defeito de fechamento da parede abdominal associado com exteriorização de estruturas intra-abdominais, principalmente o intestino fetal. O defeito é localizado na região paraumbilical, mais comumente à direita, e o cordão umbilical não apresenta alterações na sua inserção ${ }^{1}$. Raramente se associa a outras malformações ou síndromes, exceto algumas alterações intestinais como atresias ou estenoses ${ }^{2,3}$.

A incidência da gastrosquise vem aumentando nas últimas décadas em diversas populações, variando de 1 a 2 até 4 a 5/10.000 nascidos vivos, estando associada à baixa idade materna $(<20 \text { anos })^{1,4}$. Outros fatores de risco citados e sob investigação: tabagismo, drogas ilícitas e drogas vasoativas como a pseudoefedrina (dos descongestionantes nasais $)^{5,6}$. Nenhum dos fatores de risco foi ainda substancialmente comprovado com dados convincentes e reproduzidos.

A maior parte dos casos de defeitos abdominais fetais é diagnosticada no exame ultrassonográfico morfológico entre a $18^{\mathrm{a}}$ e $22^{\mathrm{a}}$ semana de gestação ${ }^{3}$. Os benefícios do diagnóstico pré-natal incluem preparo e apoio, planejamento adequado do nascimento com equipe obstétrica, cirúrgica e neonatal em alerta, categorização do risco e possibilidade de desenvolver protocolos de ação.

Nascimentos prematuros e restrição de crescimento intrauterino são esperados nos casos de gastrosquise ${ }^{7}$. Para o tratamento pós-natal, uma das maiores dificuldades para o fechamento cirúrgico primário é a desproporção visceral-abdominal. O desenvolvimento do silo foi um passo decisivo no manejo da gastrosquise. O silo consiste em uma bolsa de policloreto de polivinil (PVC) ou silicone estéril, que engloba de forma primária todo o conteúdo exteriorizado e evita a perda de calor e líquidos; progressivamente, esse conteúdo é colocado para dentro da cavidade até o fechamento cirúrgico secundário em torno de cinco a dez dias. A taxa de mortalidade atualmente gira em torno de $3,6 \%$, e os fatores mais significativos para o óbito têm sido as ressecções de intestino grosso, as anomalias congênitas circulatórias e pulmonares e a sepse ${ }^{8}$.

Este trabalho teve como objetivo analisar a taxa de mortalidade perinatal dos casos de gastrosquise atendidos em hospital de ensino do sul do Brasil e os possíveis fatores associados.

\section{Métodos}

Foi realizado um estudo de coorte retrospectivo entre 1992 e 2012. Foram incluídos todos os casos de gastrosquise nascidos no Hospital de Clínicas de Porto Alegre (HCPA), Rio Grande do Sul, Brasil, naquele período. Os autores assinaram o termo de responsabilidade para uso de dados de prontuário e a pesquisa foi aprovada pelo Comitê de Pesquisa e Ética em Saúde do grupo de pesquisa e pós-graduação do HCPA, sob o número 08-468.

As gestantes com suspeita diagnóstica atendidas no pré-natal realizado no hospital e na rede primária referenciada foram encaminhadas para o Setor de Medicina Fetal, onde realizaram exame de ultrassonografia obstétrica morfológica. O diagnóstico de gastrosquise foi definido pela confirmação ultrassonográfica do defeito da parede abdominal localizado lateralmente à inserção do cordão umbilical normalmente inserido, conteúdo abdominal flutuando no líquido amniótico, sem membrana recobrindo as estruturas ${ }^{2}$. Nos casos de suspeita, apenas no momento do nascimento, o diagnóstico foi feito pela identificação clínica do defeito.

As variáveis analisadas foram: idade materna em anos, paridade (número de partos prévios), escolaridade, peso ao nascer em gramas, idade gestacional em semanas, escore de Apgar e modo de parto (vaginal ou cesariana). Também foram analisadas as anomalias associadas encontradas (patologias, defeitos e malformações congênitas), o tipo de fechamento cirúrgico (primário ou silagem + secundário), reintervenções, sepse e outras causas do óbito. As gastrosquises foram classificadas como simples (exposição de alças intestinais somente, sem complicações) e complexas (presença de anomalias e complicações como as atresias intestinais, perfuração, necrose ou volvo) de acordo com o conteúdo eviscerado ${ }^{9,10}$. A silagem foi realizada de acordo com os protocolos hospitalares que foram sendo atualizados, inicialmente utilizando um frasco de soro estéril rígido cortado para abranger o conteúdo até o uso de bolsa de silicone de soro estéril maleável também cortada.

A mortalidade perinatal foi o desfecho principal e os casos foram divididos em mortos e vivos, sendo as variáveis comparadas entre si. Os resultados foram analisados com o programa PASW, versão 18.0, utilizando-se média e desvio-padrão para variáveis quantitativas de distribuição simétrica como peso ao nascer, pelo teste $t$ de Student, ou mediana e intervalo interquartil para variáveis quantitativas de distribuição assimétrica como 
idade materna, idade gestacional e escore de Apgar, pelo teste de Mann-Whitney. As variáveis categóricas foram analisadas utilizando-se as frequências absoluta e relativa percentuais e comparadas pelo teste do $\chi^{2}$ com correção de Yates, para as variáveis escolaridade e tipo de gastrosquise, e pelo teste exato de Fisher, para as variáveis paridade, modo de parto, anomalias associadas, tipo de fechamento cirúrgico, reintervenção e sepse. Considerou-se nível de significância de $5 \%(\mathrm{p}=0,05)$.

\section{Resultados}

Foram incluídos 64 recém-nascidos (RN) com gastrosquise; 59 deles $(92,2 \%)$ foram diagnosticados no pré-natal e os demais, ao nascimento.

As características demográficas estão descritas na Tabela 1. A maioria das gestantes com fetos com gastrosquise eram jovens (idade média de 21,5 anos), primíparas $(68,7 \%)$ e com bom nível de escolaridade $(60,9 \%$ com ensino médio e 6,3\% com ensino superior).

Foram identificadas outras anomalias associadas à gastrosquise em 21 casos: 6 casos de malrotação intestinal, 2 casos de malformações nas mãos (polegar incluso e hipoplasia de falanges), 2 casos de atresia duodenal, 2 casos de atresia de cólon, 2 casos de hidronefrose bilateral com megaureter, 1 caso de obstrução intestinal, 1 caso de criptorquidia, 1 caso de luxação congênita de quadril, 1 caso de estenose de jejuno, 1 caso de hérnia inguinal, 1 caso de teratoma ovariano associado à atresia intestinal e 1 caso de Sequência de Poland (ausência unilateral do músculo pequeno peitoral e da porção esternal do grande peitoral mais anomalia do membro superior) com dextrocardia com situs solitus (somente o coração em posição anormal à direita, os demais órgãos em posição normal).

Com relação ao conteúdo exposto, 26 casos $(40,6 \%)$ tinham o intestino somente, classificados como gastrosquises simples. Os demais tinham também o estômago em 22 casos $(34,4 \%)$ e outros órgãos em 16 casos (25\%), sendo eles: bexiga, útero, trompas, vesícula biliar, fígado, testículo, baço e ligamento redondo, classificados como gastrosquises complexas.

O fechamento cirúrgico primário foi realizado em 44 casos $(68,8 \%)$. Em 20 casos, o fechamento primário foi realizado com colocação de silo e, depois, foi realizado o fechamento cirúrgico secundário (considerados como reintervenção). Três casos de fechamento primário também necessitaram de reintervenção cirúrgica.

A mortalidade foi de $23,4 \%$ (15 mortes). Os pacientes com gastrosquise que morreram tinham peso ao nascer ( $\mathrm{p}=0,001)$, escore de Apgar $(\mathrm{p}=0,03)$ e idade gestacional ao nascimento $(\mathrm{p}=0,03)$ significativamente menor que os sobreviventes. Não houve diferença no modo de parto $(\mathrm{p}=0,8)$ e, com relação ao conteúdo eviscerado, não houve diferença entre os casos de gastrosquise simples e complexa $(\mathrm{p}=0,06)$. Também não houve diferença na mortalidade de acordo com as anomalias associadas encontradas $(\mathrm{p}=0,9)$. $\mathrm{Na}$ Tabela 2, está descrita a análise dessas variáveis do nascimento. As variáveis cirúrgicas estão descritas na Tabela 3, e a mortalidade foi significativamente maior nos casos com reintervenção $(p=0,001)$ e com sepse $(\mathrm{p}=0,008)$. Os óbitos tiveram como causa principal a seguinte descrição: 10 casos de sepse $(66,7 \%), 1$ caso de insuficiência renal aguda $(6,7 \%), 2$ casos cardiogênicos $(13,3 \%), 1$ caso de síndrome do choque tóxico $(6,7 \%)$ e 1 caso de desnutrição $(6,7 \%)$.

Tabela 1. Características maternas e demográficas dos recém-nascidos com gastrosquise

\begin{tabular}{l|c|c}
\hline Características & Valor & Indivíduos \\
\hline Idade materna (anos) & 21,5 & 64 \\
\hline Paridade (\%) & & \\
\hline Primíparas & 68,7 & 44 \\
Multíparas & 31,3 & 20 \\
\hline Escolaridade (\%) & & \\
\hline Ensino Fundamental & 26,6 & 17 \\
Ensino Médio & 60,9 & 39 \\
Ensino Superior & 6,3 & 4 \\
\hline Analfabeta & 6,3 & 4 \\
\hline Idade gestacional no nascimento (semanas) & 35,9 & 64 \\
Peso ao nascer (g) & 2.269 & 64 \\
\hline Modo de parto (\%) & & \\
\hline Cesariana & 92,2 & 59 \\
\hline Parto vaginal & 7,8 & 5 \\
\hline
\end{tabular}

Tabela 2. Variáveis do nascimento associadas à mortalidade

\begin{tabular}{|c|c|c|c|}
\hline \multirow{2}{*}{ Variáveis } & Mortos & Vivos & \multirow{2}{*}{ Valor $p$} \\
\hline & $(n=15)$ & $(n=49)$ & \\
\hline IG nascimento (semanas) & 34,5 & 36,3 & $0,03^{\star}$ \\
\hline Peso (g) & 1.900 & 2.385 & $0,001^{\star *}$ \\
\hline APGAR (média) & 4,9 & 6,8 & $0,03^{\star}$ \\
\hline \multicolumn{4}{|l|}{ Gastrosquise (\%) } \\
\hline Simples & 20 & 46,9 & $0,06^{\star \star \star}$ \\
\hline Complexa & 80 & 26 & \\
\hline \multicolumn{4}{|l|}{ Modo de parto (\%) } \\
\hline Cesariana & 93,3 & 91,8 & $0,8^{\star \star \star \star *}$ \\
\hline Parto vaginal & 6,7 & 8,2 & \\
\hline \multicolumn{4}{|l|}{ Anomalias associadas (\%) } \\
\hline Não & 66,7 & 67,3 & $0,9^{\star \star \star \star *}$ \\
\hline $\operatorname{Sim}$ & 33,3 & 32,7 & \\
\hline
\end{tabular}


Tabela 3. Distribuição percentual das variáveis cirúrgicas associadas à mortalidade

\begin{tabular}{|c|c|c|c|}
\hline \multirow{2}{*}{ Variáveis } & Mortos & Vivos & \multirow{2}{*}{ Valor p* } \\
\hline & $(n=15)$ & $(n=49)$ & \\
\hline \multicolumn{4}{|c|}{ Tipo de fechamento cirúrgico } \\
\hline Primário & 60 & 71,4 & 0,3 \\
\hline Silagem+Secundário & 40 & 28,6 & \\
\hline \multicolumn{4}{|l|}{ Reintervenção } \\
\hline Sim & 73,3 & 24,5 & 0,001 \\
\hline Não & 26,7 & 75,5 & \\
\hline \multicolumn{4}{|l|}{ Sepse } \\
\hline Sim & 80 & 40,8 & 0,008 \\
\hline Não & 20 & 59,2 & \\
\hline
\end{tabular}

*Teste exato de Fisher.

\section{Discussão}

A taxa de prevalência de gastrosquise tem aumentado progressivamente em todas as regiões do mundo; costumava ser de 1:50.000 nascimentos próximo à década de 1960, quando se iniciaram os programas de vigilância e coletas de dados sobre malformações ao nascimento no mundo. Desde então, tem aumentado, chegando a cerca de 10 a 15 vezes em várias populações. Segundo dados do Estudo Colaborativo Latino-Americano de Malformações Congênitas (ECLAMC), a prevalência na América do Sul é de 2,9:10.000 1,4 . A taxa de Porto Alegre entre os anos de 2000 e 2004 foi de 4,53:10.000 nascidos vivos e, no HCPA, foi de 5,42:10.000 nascidos vivos (dados do programa local de monitoramento de defeitos congênitos, associado ao ECLAMC).

Muitos autores têm relatado o aumento das taxas de sobrevivência entre os pacientes com gastrosquise nas últimas décadas, entre 90 a $95 \%$ nos países desenvolvidos ${ }^{1,3-5,8,11}$. No Brasil, a mortalidade varia de acordo com a região; no Nordeste, chega a $52 \%{ }^{12,13}$. Em nosso estudo, a taxa foi de $23,4 \%$, ainda mais alta do que nos países desenvolvidos, mas menor do que em outras regiões brasileiras.

Com relação aos fatores associados à mortalidade, ainda o baixo peso e a prematuridade, que foram significativos no nosso estudo, são causas pertinentes e não especificamente associadas à gastrosquise. Diretamente ligada à patologia, a complexidade é um fator específico. Desordens intestinais, tais como a atresia e o volvo, e as complicações como a enterocolite necrotizante e a perfuração intestinal parecem aumentar a mortalidade e a morbidez dos pacientes com gastrosquise ${ }^{8-10,14,15}$. A dilatação intra-abdominal intestinal é um dos poucos marcadores de complexidade da gastrosquise que podem ser identificados na ultrassonografia pré-natal, podendo ser utilizada na estratificação de risco ${ }^{16}$. Além dela, a presença de vários órgãos eviscerados além do intestino também acrescenta complexidade à gastrosquise e ao seu tratamento cirúrgico, conforme o volume do conteúdo exteriorizado. Em nossa coorte, na análise do tipo de gastrosquise, a maioria dos óbitos ocorreu em pacientes com gastrosquise complexa, embora não tenha havido diferença estatística, provavelmente associada ao número dos casos de óbito.

Não encontramos associação entre o tipo de parto e a mortalidade. Outros autores também não encontraram benefício com a cesariana de rotina, e o tipo ideal de parto ainda permanece controverso ${ }^{2,7,15,17}$. A indicação da via de parto tem sido a cesariana para pacientes com diagnóstico pré-natal estabelecido no HCPA para planejar o momento ideal de intervenção cirúrgica pela equipe da cirurgia pediátrica (disponibilidade de sala cirúrgica e anestesista). Como hospital de referência, muitos casos de gastrosquise nascidos em outros hospitais ou cidades acabam sendo transferidos para o HCPA; não avaliamos os casos nascidos fora do hospital neste trabalho, mas já foi demonstrado pelo estudo de Vilela no Nordeste do Brasil uma mortalidade maior em seus casos transferidos, o que poderia estar relacionado ao manejo sub-ótimo e às condições de transporte em muitas cidades daquela região $0^{13}$.

A análise dos resultados de acordo com o tipo de fechamento cirúrgico não revelou diferenças significativas, o que está de acordo com resultados encontrados na literatura ${ }^{3,7,18}$. O tratamento de escolha da gastrosquise tem sido extensamente debatido, mas as evidências disponíveis são bastante heterogêneas. As complicações das reintervenções realizadas tanto para o fechamento secundário como após o fechamento primário e a sepse estão associadas com altas taxas de mortalidade, sendo a última responsável por $66,7 \%$ das mortes, o que foi similar à encontrada em outros estudos brasileiros ${ }^{12,13}$. Medidas podem ser tomadas para minimizar tal complicação e posterior redução dessas taxas. Um estudo recente revisando o manejo de gestações com gastrosquise concluiu que a indução de parto na $37^{\mathrm{a}}$ semana de gestação esteve associada com redução da sepse e morte neonatal em comparação com o manejo expectante, embora revisão feita pela Cochrane não tenha sido capaz de afirmar tal conclusão ${ }^{19,20}$. A estratificação do risco parece ser possível e pode ser utilizada em nossa realidade, mas algumas questões ainda necessitam ser respondidas, como o planejamento do nascimento conforme o diagnóstico da complexidade da gastrosquise e cobertura antibiótica específica para prevenção das complicações da sepse $e^{9,10,15,16}$. A longa permanência no hospital e as complicações gastrointestinais são fatores a serem esclarecidos para as famílias dos RN com gastrosquise ${ }^{21}$. Protocolos estão sendo desenvolvidos para padronizar as condutas, técnicas cirúrgicas e coleta de dados para informação e estudos, iniciados no ano de 2013, incentivados pelos resultados do presente estudo. Como o trabalho foi feito com a análise dos dados dos prontuários de até 20 anos 
atrás, algumas informações importantes podem ter sido omitidas, sendo uma de suas limitações.

A mortalidade perinatal da gastrosquise neste trabalho parece depender principalmente da prematuridade, baixo peso e complicações cirúrgicas. Os benefícios do diagnóstico pré-natal da gastrosquise são muitos e incluem preparo e apoio familiar, planejamento adequado do nascimento com equipes obstétrica, cirúrgica e neonatal em alerta, categorização do risco e possibilidade de desenvolver protocolos específicos de ação, com o objetivo de reduzir ao máximo as taxas de mortalidade perinatal associadas a essa doença.

\section{Referências}

1. Castilla EE, Mastroiacovo P, Orioli IM. Gastroschisis: international epidemiology and public health perspectives. Am J Med Genet C Semin Med Genet. 2008;148C(3):162-79.

2. Ledbetter DJ. Gastroschisis and omphalocele. Surg Clin North Am. 2006;86(2):249-60

3. Nichol PF, Byrne JL, Dodgion C, Saijoh Y. Clinical considerations in gastroschisis: incremental advances against a congenital anomaly with severe secondary effects. Am J Med Genet C Semin Med Genet. 2008; 148C(3):231-40.

4. Santiago-Munoz PC, Mclntire DD, Barber RG, Megison SM, Twicler DM, Dashe JS. Outcomes of pregnancies with fetal gastroschisis. Obstet Gynecol. 2007; 1 10(3):663-8.

5. Rasmussen SA, Frías JL. Non-genetic risk factors for gastroschisis. Am J Med Genet C Semin Med Genet. 2008;148C(3):199-212.

6. Feldkamp ML, Botto LD. Developing a research and public health agenda for gastroschisis: how do we bridge the gap between what is know and what is not? Am J Med Genet C Semin Med Genet. 2008; 148C(3):155-61.

7. Hunter AG, Stevenson RE. Gastroschisis: clinical presentation and associations. Am J Med Genet C Semin Med Genet. 2008; 148C(3):219-30.

8. Lao $O B$, Larison $C$, Garrison MM, Waldhausen JH, Goldin AB. Outcomes in neonates with gastroschisis in US children's hospitals. Am J Perinatol. 2010;27(1):97-101

9. Arnold MA, Chang DC, Nabaweesi R, Colombani PM, Bathurst MA, Mon KS, et al. Risk stratification of 4344 patients with gastroschisis into simple and complex categories. J Pediatr Surg. 2007;42(9): 1520-5

10. Molik KA, Gingalewski CA, West KW, Rescorla FJ, Scherer LR, Engum SA, et al. Gastroschisis: a plea for risk categorization. J Pediatr Surg. 2001;36(1):51-5.
1 1. Curry Jl, McKinney P, Thornton JG, Stringer MD. The aetiology of gastroschisis. BJOG. 2000;107(11):1339-46.

12. Amorim MMR, Vilela PC, Santos LC, Falbo Neto GH, Lippo LAM, Marques M. [Gastroschisis: prenatal diagnosis $\times$ neonatal outcome.] Rev Bras Ginecol Obstet. 2000;22(4):191-9. Portuguese.

13. Vilela PC, Amorim MMR, Falbo GH, Santos LC. Risk factors for adverse outcome of newborns with gastroschisis in a Brazilian hospital. J Pediatr Surg. 2001;36(4):559-64.

14. Logghe HL, Mason GC, Thornton JG, Stringer MD. A randomized controlled trial of elective preterm delivery of fetuses with gastroschisis. J Pediatr Surg. 2005;40(1 1):1726-31

15. Arnold MA, Chang DC, Nabaweesi R, Colombani PM, Fischer AC, Lau HT, et al. Development and validation of a risk stratification index to predict death in gastroschisis. J Pediatr Surg. 2007;42(9):950-5.

16. Kuleva M, Khen-Dunlop N, Dumez Y, Ville Y, Salomon LJ. Is complex gastroschisis predictable by prenatal ultrasound? BJOG. 2012;119(1):102-9.

17. Jager LC, Heii HA. Factors determining outcome in gastroschisis: clinical experience over 18 years. Pediatr Surg Int. 2007;23(8):731-6.

18. Reigstad I, Reigstad H, Kiserud T, Berstad T. Preterm elective caesarean section and early enteral feeding in gastroschisis. Acta Paediatr. $2011 ; 100(1): 71-4$.

19. Baud D, Lausman A, Alfaraj MA, Seaward G, Kingdom J, Windrim $R$, et al. Expectant management compared with elective delivery at 37 weeks for gastroschisis. Obstet Gynecol. 2013;121 (5):990-8.

20. Grant NH, Dorling J, Thornton JG. Elective preterm birth for fetal gastroschisis. Cochrane Database Syst Rev. 2013;6:CD009394.

21. Durfee $S M$, Benson $C B$, Adams SR, Ecker J, House M, Jennings $R$, et al. Postnatal outcome of fetuses with the prenatal diagnosis of gastroschisis. J Ultrasound Med. 2013;32(3):407-12. 